\title{
INTERDISCIPLINA Y EDUCACIÓN: LA ORIENTACIÓN DEPORTIVA COMO PROPUESTA SISTÉMICA
}

\section{INTERDISCIPLINE AND EDUCATION: THE SPORTIVE ORIENTEERING AS SYSTEMIC PROPOSAL}

\author{
Franklin Castillo-Retamal ${ }^{1}$, Fernanda Cordero-Tapia ${ }^{1}$, Allana Joyce Gomes Soares \\ Scopel ${ }^{2}$ \\ fcastillo@ucm.cl \\ fdacorderotapia@gmail.com \\ allana@ifce.edu.br \\ ${ }^{1}$ Universidad Católica del Maule \\ ${ }^{2}$ Instituto Federal de Educación, Ciencia y Tecnología de Ceará, Brasil
}

Envío Original: 2019-03-22 Reenviado: 2019-07-12 Aceptado: 2019-09-26

Publicado: 2019-10-23

Doi: https://doi.org/10.15517/pensarmov.v17i2.36693

\begin{abstract}
Resumen
Se presentan resultados de una búsqueda bibliográfica basada en interrogantes relacionadas a las posibilidades de la práctica de la orientación deportiva como componente curricular pertinente en las aulas escolares. Se adoptó un trabajo cualitativo de naturaleza básica tratando de generar conocimientos nuevos y útiles sin necesidad de una aplicación práctica inmediata (Engel y Tolfo, 2009), teniendo como procedimiento la investigación bibliográfica, que, según Silva y Muszkat (2005), es aquella elaborada a partir de material publicado. Se concluye que el desarrollo de actividades en el medio natural es beneficioso para la formación integral del educando y, la orientación deportiva, es un deporte que promueve el desarrollo de habilidades y conductas asociadas al entendimiento holístico del fenómeno humano a partir de la integración de saberes.
\end{abstract}

Palabras clave: interdisciplinariedad, currículo, escuela, deporte.

\begin{abstract}
The results of a literature search based on the questions related to the possibilities of the practice of sportive orienteering as relevant curricular component in school classrooms are presented. A qualitative work of a basic nature was adopted trying to generate new and useful knowledge without needing an immediate practical application (Engel \& Tolfo, 2009), having as a procedure the bibliographic research, that, according to Silva \& Muszkat (2005), is that elaborated from published material. It is concluded that the development of activities
\end{abstract}


in the natural environment is beneficial for the integral education of the student and, the sportive orienteering, is a sport that promotes the development of skills and behaviors associated with the holistic understanding of the human phenomenon from the integration of knowledge.

Keywords: interdisciplinary, curriculum, school, sport.

\section{Introducción}

La orientación es una herramienta que tiene posibilidades de transferencia en la vida diaria, puesto que orientarse forma parte de nuestro quehacer rutinario, aunque no nos demos cuenta. Nos sirve para interpretar planos de una ciudad, un terreno, conocer y reconocer nuevos espacios, además de adaptarnos a nuevos lugares con prontitud y autonomía. Junto con eso, facilita encontrar lugares en sitios desconocidos, ayuda a calcular distancias y tiempos, reduce el riesgo de perderse o extraviarse y garantiza una progresión más segura (Julián y Pinos, 2011; Pimentel et al., 2017).

En este contexto, la orientación favorecería el contacto con la naturaleza además de contribuir en la prevención del sedentarismo, el desarrollo de la concentración y, junto con eso, enfermedades alusivas al sobrepeso. Según Freire (2011), el principal objetivo de los niños de 5 a 12 años es descubrir su entorno, explorando los alrededores de su vivienda y escuela, su pueblo y región, incorporando a través de estas actividades una comprensión intuitiva de conceptos abstractos de la física, las matemáticas y el medio ambiente.

El medio natural entrega la posibilidad de generar vivencias significativas para los estudiantes y, según Wells (2000) y Castillo (2006), el estar en entornos naturales mejora la capacidad cognitiva además de aumentar la concentración en los niños. En este sentido Kellert (2005), Fuentealba, Marín, Castillo y Roco (2017), indican la importancia de la naturaleza desde el aspecto intelectual, emocional, social, espiritual y físico, como un espacio propicio para adquirir conocimientos únicos que favorecen el aprendizaje.

Estudios realizados en los patios de las escuelas, evidencian que los niños participan en juegos más creativos y de forma más cooperativa cuando se encuentran en áreas verdes (Bell y Dyment, 2006). Desde esta perspectiva, podría inferirse que la orientación deportiva permitiría al estudiante desarrollar no solo el aspecto motriz, sino que también favorecería el desarrollo cognitivo, emocional y social, junto con hacerle consciente respecto del entorno que lo rodea.

Al considerar la orientación como un elemento de relevancia en el desarrollo integral de las personas, es posible mencionar los clásicos principios generales planteados por Miller (1971), en los que propone que es para todos y de todas las edades, debe abordar todos los aspectos del desarrollo, como así mismo, incentivar el descubrimiento y desarrollo personal, junto con eso, debe ser una tarea colaborativa en la que se comprometa al estudiante, profesores y agentes directivos del centro educativo.

El propósito del estudio se centra en la observación de las posibilidades que presenta la orientación deportiva teniendo como base su flexibilidad y aplicabilidad en distintos espacios y contextos. Tomando en consideración todos los antecedentes, el trabajo atiende 
a lo menos dos objetivos: 1) establecer una línea de desarrollo basada en la orientación deportiva como catalizador para el trabajo interdisciplinar; 2) proponer un abordaje metodológico interdisciplinar orientado al contexto escolar. Para alcanzar estos objetivos, se utilizó la metodología cualitativa básica, que en su más amplio sentido intenta generar conocimientos nuevos y útiles sin una inmediata aplicación práctica (Engel y Tolfo, 2009). Desde el punto de vista procedimental, se trata de una investigación descriptiva, adoptándose la técnica de investigación bibliográfica, entendida como aquella elaborada a partir de material publicado (Silva y Muszkat, 2005). Los datos obtenidos corresponden a la categoría de secundarios y fueron analizados a partir de lo propuesto por Bardin (2011), respecto al análisis de contenido, cuyas fases son pre-análisis, exploración del material y tratamiento de los resultados, inferencia e interpretación. El tipo de muestra utilizado responde, según McMillan y Schumacher (2005), al muestreo intencionado, toda vez que se ha seleccionado información detallada con el fin de entender algo sin necesidad de generalizar y con la intención de aumentar la utilidad de la misma. Al mismo tiempo, la estrategia utilizada para fomentar la validez de los datos fue una de las diez indicadas por McMillan y Schumacher (2005), en este caso, la de investigadores múltiples, que en su despliegue coincide con los datos recogidos a partir de algunas de sus investigaciones. La subjetividad de los autores ha sido controlada a partir de lo planteado por Marimon y Martos (2014), en relación a los principios de la descripción objetiva, entendida como el tratamiento textual con un léxico preciso y riguroso y, el punto de referencia, asociado al fenómeno desde el cual se evalúa una condición o cualidad.

\section{Interdisciplina - Deporte - Educación: la triada integradora}

El término interdisciplinariedad, acuñado por el sociólogo Louis Wirtzy en 1937 (Lenoir, 1999), es la cualidad de interdisciplinario, aquello que se realiza con la cooperación de varias disciplinas (Díaz, 2010). Actualmente, es un concepto posible de trabajar desde diferentes ámbitos como el social, cultural, personal, medioambiental, deportivo y educativo, por lo que las temáticas interdisciplinares son aquellas que se desarrollan de manera colaborativa entre dos o más áreas que persiguen un objetivo específico y el desarrollo integral del individuo.

Encontramos en la literatura diferentes definiciones de interdisciplinariedad y cada una de ellas asume las especificidades del contexto en que son usadas (Tabla 1). 
Tabla 1

Conceptualización de interdisciplinariedad en las últimas dos décadas.

\begin{tabular}{|c|c|c|}
\hline Autor & Año & Definición \\
\hline Torres & $\underline{1996}$ & $\begin{array}{l}\text { Elaboración de marcos conceptuales más generales, en los } \\
\text { cuales las diferentes disciplinas en contacto son a la vez } \\
\text { modificadas y pasan a depender unas de otras. }\end{array}$ \\
\hline Posada & $\underline{2004}$ & $\begin{array}{l}\text { Es el segundo nivel de integración disciplinar, en el cual la } \\
\text { cooperación entre disciplinas conlleva interacciones reales, es } \\
\text { decir, reciprocidad en los intercambios y, por consiguiente, un } \\
\text { enriquecimiento mutuo. }\end{array}$ \\
\hline Van del Linde & $\underline{2007}$ & $\begin{array}{l}\text { Puede verse como una estrategia pedagógica que implica la } \\
\text { interacción de varias disciplinas, entendida como el diálogo y } \\
\text { la colaboración de éstas para lograr la meta de un nuevo } \\
\text { conocimiento. }\end{array}$ \\
\hline Carvajal & $\underline{2010}$ & $\begin{array}{l}\text { Permite generar un pensamiento flexible, desarrolla y mejora } \\
\text { habilidades de aprendizaje, facilita el entendimiento, } \\
\text { incrementa la habilidad de acceder al conocimiento adquirido } \\
\text { y mejora las habilidades para integrar contextos disímiles. }\end{array}$ \\
\hline $\begin{array}{l}\text { Rivero, López-Huertas y } \\
\text { Pérez }\end{array}$ & $\underline{2013}$ & $\begin{array}{l}\text { Es un enfoque ensamblado, donde las disciplinas tienden a } \\
\text { mezclarse en sus fronteras. }\end{array}$ \\
\hline Llano et al. & $\underline{2016}$ & $\begin{array}{l}\text { Es el resultado del desarrollo de la ciencia y la tecnología, así } \\
\text { como de las conexiones, interacciones, fusiones e integración } \\
\text { de los diversos planos de la vida humana. }\end{array}$ \\
\hline Sotolongo y Delgado & $\underline{2016}$ & $\begin{array}{l}\text { Es el esfuerzo indagatorio y convergente entre varias } \\
\text { disciplinas (en ese sentido, presupone la multidisciplinariedad) } \\
\text { pero que persigue el objetivo de obtener "cuotas de saber" } \\
\text { acerca de un objeto de estudio nuevo, diferente a los que } \\
\text { pudieran estar previamente delimitados, disciplinaria o } \\
\text { multidisciplinariamente. }\end{array}$ \\
\hline Henao et al. & $\underline{2017}$ & $\begin{array}{l}\text { Integración de disciplinas con cierta afinidad en su orientación } \\
\text { hacia el objeto de estudio. }\end{array}$ \\
\hline $\begin{array}{l}\text { Longchamp-Ges, } \\
\text { Escalona-Vásquez y } \\
\text { Céspedes-Quiala }\end{array}$ & $\underline{2018}$ & $\begin{array}{l}\text { Establecimiento de nexos recíprocos, interacciones, } \\
\text { intercambios múltiples y cooperación entre las ciencias que } \\
\text { tienen en común un objeto de estudio. }\end{array}$ \\
\hline $\begin{array}{l}\text { Paula-Acosta, Pérez- } \\
\text { López y Sierra-Socorro }\end{array}$ & $\underline{2019}$ & $\begin{array}{l}\text { Trabajo metodológico cooperado como vía para la integración } \\
\text { de los saberes }\end{array}$ \\
\hline
\end{tabular}

Fuente: elaboración de los autores. 
A partir de estos supuestos y entendiendo el carácter sistémico del proceso educativo, la interdisciplinariedad en este espacio la entenderemos como el punto de comunión que entrega una visión amplia del fenómeno desde la integración de la multidimensionalidad del conocimiento y, por ende, del propio ser humano. Vale decir, esta forma de comprender la realidad desde la globalidad, permitiría al ser humano conocerse y sentirse parte del mundo al percibir su entorno desde la totalidad y complejidad, logrando de esa manera aprendizajes con sentido, en palabras de Varela (2000), "nos encontramos con un ser indivisible que no puede ser trabajado o desarrollado por partes sino que, por el contrario, cada parte actualiza el conjunto y el conjunto actualiza la parte", o sea, la parcelación del conocimiento nos lleva a una visión fragmentada del mundo, que a su vez es una trama que debemos entender en su totalidad y complejidad (Castillo, 2010).

La interdisciplinariedad cobra sentido en la medida en que flexibiliza y amplía los marcos de referencia de la realidad a partir de la permeabilidad entre las verdades de cada uno de los saberes (Follari, 2007), por lo que es uno de los campos que en educación supone colaboración entre especialistas para romper las líneas divisorias de las disciplinas.

En este contexto, la orientación deportiva es adaptable y aplicable en el aula de manera interdisciplinar y también como un eje trasversal, toda vez que nos ofrece la posibilidad de abordar más de una asignatura o materia del currículo escolar mediante su práctica, donde es posible integrar diferentes habilidades a partir de las distintas áreas de aprendizaje (Escaravajal-Rodríguez, 2017). Desde esta perspectiva, favorece la formación integral del individuo, lo que conlleva una serie de beneficios a partir de lo conceptual, procedimental y actitudinal (Sicilia y Rivadeneira (1998), McNeill, Cory-Wrigth y Renirew (2006), Castillo y Cordero (2017). En la Figura 1, hay una representación de cómo la orientación puede ser abordada en el contexto escolar desde una perspectiva holística: 


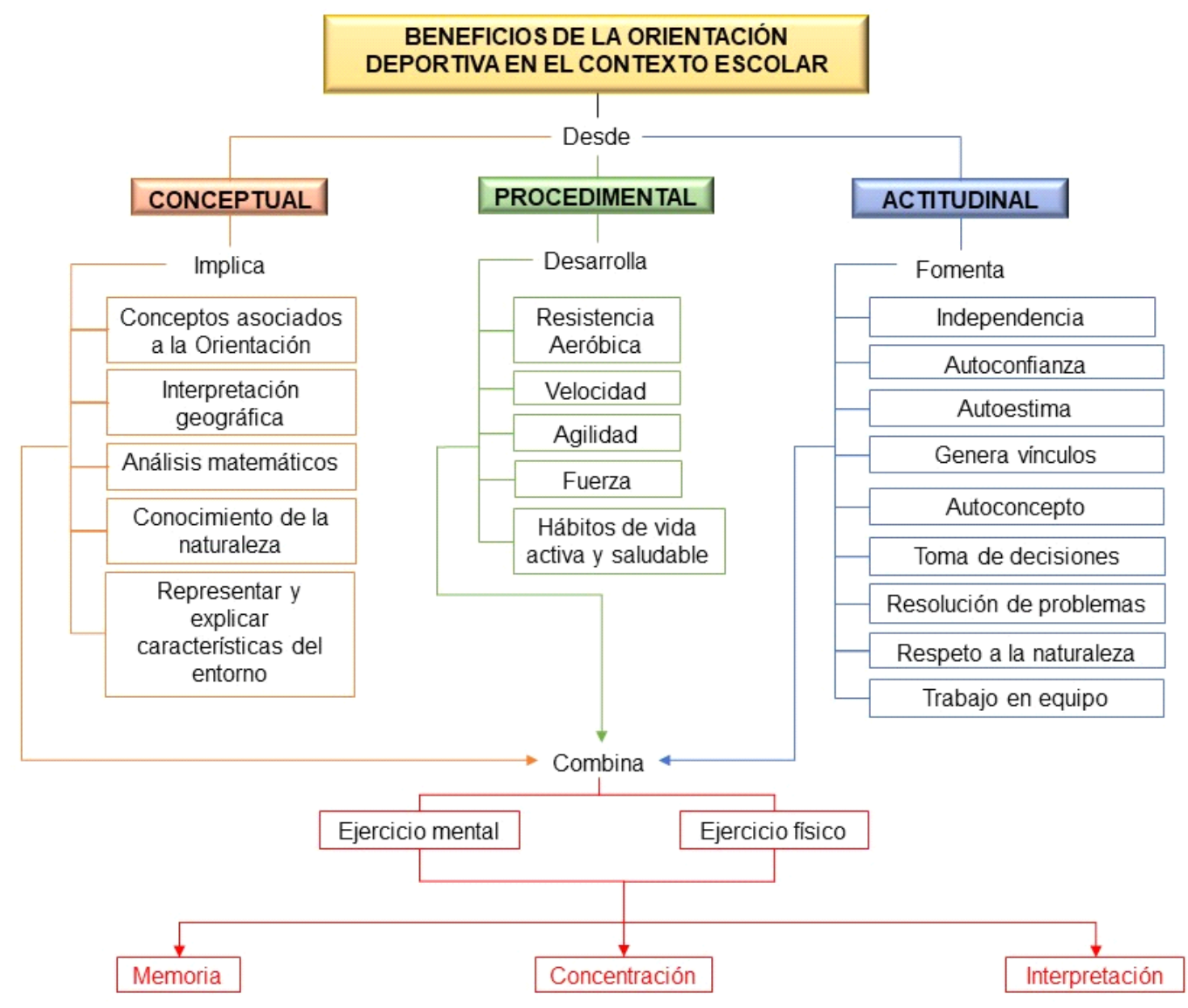

Figura 1. Beneficios de la orientación deportiva en el contexto escolar. Fuente: Elaboración de los autores, adaptado de Sicilia y Rivadeneira (1998), McNeill, CoryWrigth y Renirew (2006), Castillo y Cordero (2017).

Además de estos beneficios, es posible que, a partir de lo procedimental, se contribuya para una buena comprensión de la lateralidad, el desarrollo de la coordinación motora y otras habilidades de locomoción y estabilidad, especialmente cuando su práctica se inicia en los primeros años de educación básica.

Cuando nos referimos a la orientación deportiva, estamos hablando de una alternativa que permite, entre otras cosas:

- Cifrar y descifrar información

- Identificar debilidades

- Saber actuar en función de las propias posibilidades

- Elaborar estrategias

- Conocer y respetar el medio ambiente

- Fomentar la educación en valores

- Manejar situaciones frente a la adversidad

- Enfrentarse a situaciones difíciles 
A partir de esto, es posible indicar que la orientación deportiva es un contenido educativo transversal e interdisciplinar (Muñoz, 2010; Pimentel et al., 2017) pudiéndose transformar en una actividad o estrategia viable para implementar en las aulas, toda vez que se trabaje y entienda de manera integral.

\section{Propuesta de progresión de la orientación en la escuela}

La orientación en la escuela permite reforzar aspectos éticos, valóricos y sociales (Pimentel et al., 2017) y, una de las ventajas, es que los materiales a utilizar para su práctica son económicos y de rápida generación, lo que significa una inserción más fácil en las aulas.

Para su enseñanza, se requiere un trabajo metodológico que implica ir desde lo más básico a lo más complejo. En esta línea, se plantean tres niveles de aprendizaje:

- Descubrimiento: en esta primera etapa, el estudiante reconoce los entornos más próximos, lo que permite la comprensión de su realidad corporal y espacial desde las características que este posee, realizando gráficas del mismo de tal forma de adquirir nociones básicas sobre el concepto de orientación.

- Dominio: en esta etapa, el estudiante interpreta símbolos, hace lectura de los elementos del entorno incorporando recorridos cortos y básicos con la utilización de un mapa.

- Técnica: aquí, el estudiante aplica técnicas físicas, manejo de instrumentos y técnicas cognitivas, lo que implica desplazarse de manera autónoma realizando lecturas más detalladas en diferentes entornos.

En la Figura 2, tenemos una representación de cómo es posible enseñar la orientación considerando estos tres niveles de aprendizaje: 


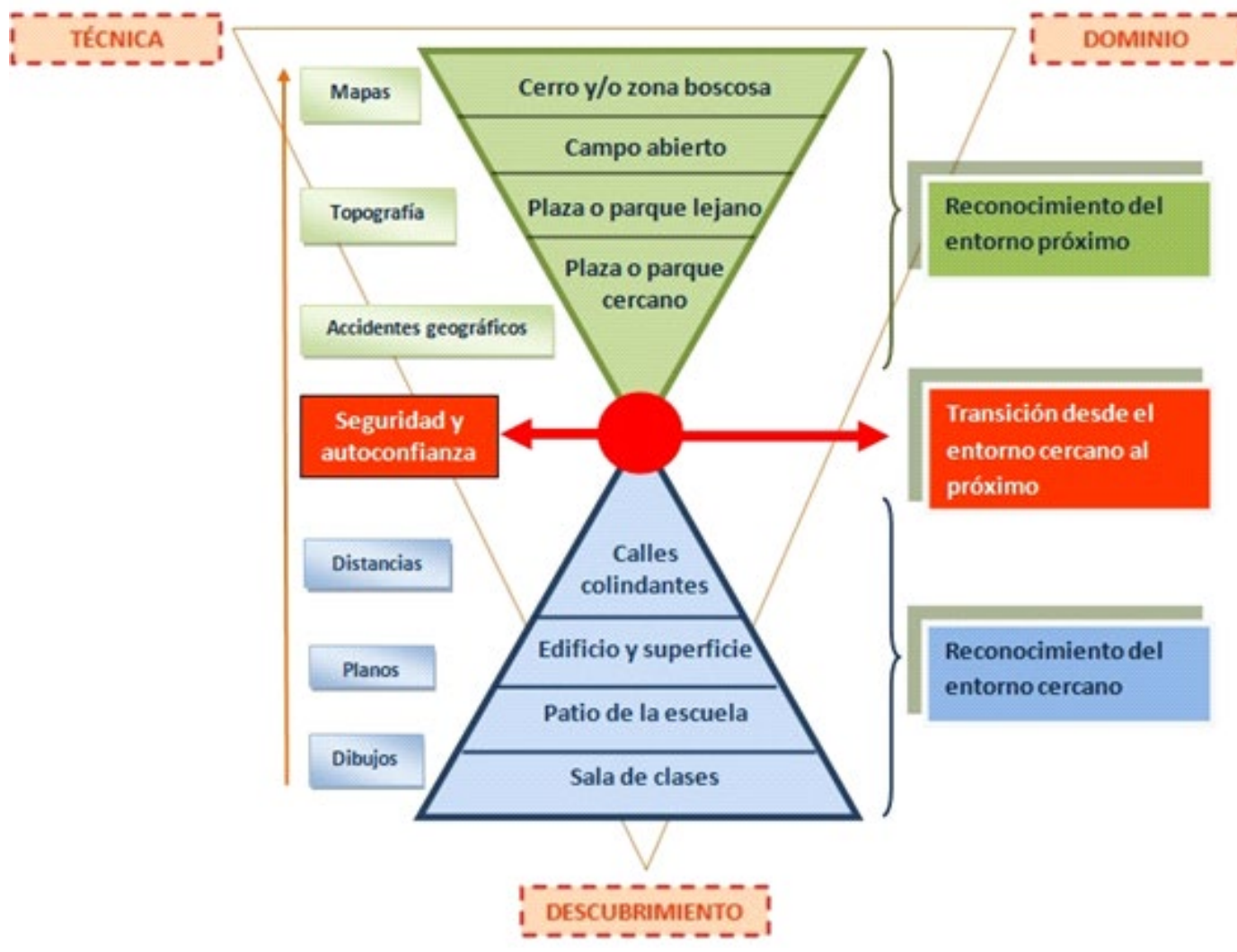

Figura 2. Progresión espacial para el trabajo de orientación en la escuela. Fuente: Elaboración de los autores.

Este proceso de aprendizaje debe ser abordado desde un enfoque elemental hasta alcanzar un nivel avanzado. A partir de cada nivel de aprendizaje, se proponen las siguientes categorías para su aplicación:

- Desde lo cognitivo: corresponde a la adquisición de los conocimientos relacionados con los contenidos teóricos asociados a cada uno de los niveles de aprendizaje en la orientación.

- Desde lo afectivo y emocional: corresponde al fomento de una educación en valores mediante la adquisición y aplicación de los contenidos durante el proceso de enseñanza, fortaleciendo el manejo de las emociones frente a situaciones asociadas a su práctica.

- Desde lo motriz: corresponde al proceso de aplicación de los contenidos teóricos asociados a cada uno de los niveles de aprendizaje en la orientación.

- Desde el medio: corresponde al lugar espacial en el que se llevan a cabo las acciones pedagógicas para el aprendizaje de los contenidos.

- Situaciones pedagógicas: corresponde al conjunto de acciones planificadas para desarrollar dentro o fuera del aula, de carácter individual o grupal, que tienen como propósito alcanzar los objetivos y finalidades de la enseñanza (ámbitos cognitivo, motriz y afectivo). 
A modo de resumen, se presenta una progresión metodológica para el trabajo de orientación en la escuela a partir de los niveles de aprendizaje:

- $\quad$ Nivel de Descubrimiento

\begin{tabular}{|c|c|c|c|c|}
\hline Desde lo cognitivo & $\begin{array}{c}\text { Desde lo afectivo y } \\
\text { emocional }\end{array}$ & Desde lo motriz & $\begin{array}{l}\text { Desde el } \\
\text { medio }\end{array}$ & $\begin{array}{l}\text { Situaciones } \\
\text { pedagógicas }\end{array}$ \\
\hline $\begin{array}{l}\text {-Reconocimiento de } \\
\text { cartas y planos } \\
\text {-Relación } \\
\text { carta/terreno } \\
\text {-Uso de brújula }\end{array}$ & $\begin{array}{l}\text {-Autoconfianza } \\
\text {-Trabajo en equipo } \\
\text {-Apoyo entre } \\
\text { compañeros } \\
\text {-Solidaridad }\end{array}$ & $\begin{array}{l}\text {-Desarrollo del } \\
\text { ritmo de carrera } \\
\text {-Desarrollo de la } \\
\text { resistencia } \\
\text { aeróbica, fuerza y } \\
\text { velocidad } \\
\text {-Carreras cortas }\end{array}$ & $\begin{array}{l}\text {-Sala de clase } \\
\text {-Patio del } \\
\text { establecimiento }\end{array}$ & $\begin{array}{l}\text {-Dibujar planos } \\
\text {-Observar planos } \\
\text { con relación al } \\
\text { terreno } \\
\text {-Circuitos guiados } \\
\text {-Identificación de } \\
\text { curvas de nivel }\end{array}$ \\
\hline
\end{tabular}

- Nivel de Dominio

\begin{tabular}{|c|c|c|c|c|}
\hline $\begin{array}{l}\text { Desde lo } \\
\text { cognitivo }\end{array}$ & $\begin{array}{c}\text { Desde lo afectivo y } \\
\text { emocional }\end{array}$ & Desde lo motriz & Desde el medio & $\begin{array}{l}\text { Situaciones } \\
\text { pedagógicas }\end{array}$ \\
\hline $\begin{array}{l}\text {-Interpretar } \\
\text { símbolos } \\
\text {-Interpretar carta } \\
\text { topográfica } \\
\text {-Manejo de la } \\
\text { brújula }\end{array}$ & $\begin{array}{l}\text {-Toma de decisiones } \\
\text {-Confianza } \\
\text {-Concentración } \\
\text {-Respeto a la } \\
\text { naturaleza y } \\
\text { compañeros }\end{array}$ & $\begin{array}{l}\text {-Carrera } \\
\text { cronometrada } \\
\text {-Azimut y retro- } \\
\text { azimut }\end{array}$ & $\begin{array}{l}\text {-Plazas cercanas } \\
\text { al establecimiento } \\
\text {-Bosques }\end{array}$ & $\begin{array}{l}\text {-Circuito estrella } \\
\text {-Calcular distancia } \\
\text {-Calcular grados } \\
\text {-Búsqueda de } \\
\text { controles en el } \\
\text { gimnasio o espacio } \\
\text { abierto }\end{array}$ \\
\hline
\end{tabular}

- $\quad$ Nivel Técnico

\begin{tabular}{|c|c|c|c|c|}
\hline $\begin{array}{l}\text { Desde lo } \\
\text { cognitivo }\end{array}$ & $\begin{array}{c}\text { Desde lo afectivo y } \\
\text { emocional }\end{array}$ & $\begin{array}{c}\text { Desde lo } \\
\text { motriz }\end{array}$ & Desde el medio & $\begin{array}{l}\text { Situaciones } \\
\text { pedagógicas }\end{array}$ \\
\hline -Navegación & $\begin{array}{l}\text {-Autonomía } \\
\text {-Seguridad en la ruta } \\
\text {-Honestidad }\end{array}$ & $\begin{array}{l}\text {-Carreras de } \\
\text { larga distancia } \\
\text {-Carrera de } \\
\text { media distancia } \\
\text {-Carrera de } \\
\text { sprint }\end{array}$ & $\begin{array}{l}\text {-Espacios } \\
\text { urbanos } \\
\text {-Espacios } \\
\text { naturales } \\
\text { artificializados } \\
\text {-Espacios } \\
\text { naturales } \\
\text { desconocidos }\end{array}$ & $\begin{array}{l}\text {-Trabajo de relevos } \\
\text {-Tomar rumbo con la } \\
\text { brújula } \\
\text {-Lectura de mapas } \\
\text {-Recorridos en } \\
\text { diferentes lugares }\end{array}$ \\
\hline
\end{tabular}

Como plantea McNeill et al. (2006), al desarrollar la orientación se trabajan habilidades físicas y cognitivas, los estudiantes disfrutan de las emociones que se generan a partir de esta práctica junto con vivenciar una buena navegación. En este punto, es 
importante comprender que esta progresión metodológica es solo una forma de representación de los contenidos que podrían ser enseñados en cada categoría de aplicación de acuerdo con los niveles de aprendizaje, de tal manera que no deben ser abordados de forma fragmentada, sino que estableciendo las relaciones de interdependencia entre los mismos.

En el sistema educativo, tomando como referencia las Bases Curriculares para la Educación Básica en Chile (Ministerio de Educación del Gobierno de Chile, 2012), es posible vincular la orientación deportiva a las siguientes asignaturas:

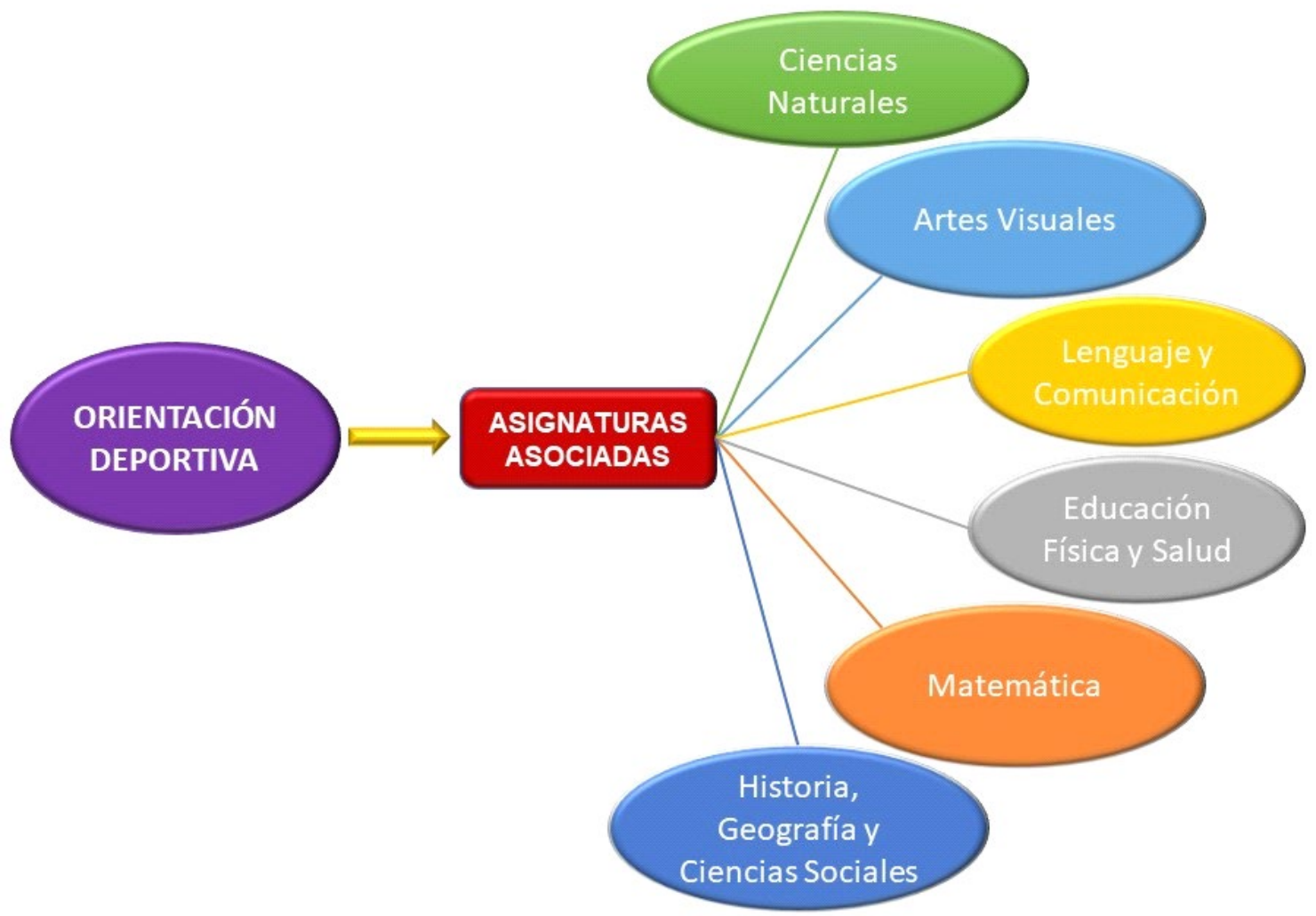

Figura 3. Orientación deportiva asociada a las asignaturas del curriculum escolar. Fuente: Elaboración de los autores. 
En este sentido, se sugiere la siguiente vinculación aplicable al contexto escolar (Tabla 2):

Tabla 2

Orientación deportiva asociada a los contenidos de las asignaturas del currículo escolar.

\begin{tabular}{|c|c|}
\hline Asignatura & Orientación deportiva \\
\hline $\begin{array}{l}\text { Ciencias } \\
\text { Naturales }\end{array}$ & $\begin{array}{l}\text {-Movimientos de la Tierra. } \\
\text {-Características del medio. } \\
\text {-Observación de elementos del medio físico. } \\
\text {-Medio Ambiente. }\end{array}$ \\
\hline Artes Visuales & $\begin{array}{l}\text {-Representar en un plano las distancias, recorridos y situación de objetos. } \\
\text {-Interpretar el contenido de imágenes y representaciones. } \\
\text {-Representar las formas en el espacio. } \\
\text {-Percepción visual frente a diferentes paisajes. }\end{array}$ \\
\hline $\begin{array}{l}\text { Lenguaje y } \\
\text { Comunicación }\end{array}$ & $\begin{array}{l}\text {-Interpretar, expresar y representar hechos, conceptos y procesos del medio natural. } \\
\text {-Interpretar símbolos. } \\
\text {-Desarrollo personal a partir de actividades de participación colectiva. } \\
\text {-Interpretación y descripción verbal de croquis de itinerarios y elaboración de los mismos. } \\
\text {-Descripción de la posición de objetos del entorno respecto de sí mismo: delante/detrás } \\
\text { de mí, encima/debajo de mí, a mi derecha/ izquierda. } \\
\text {-Describir el desplazamiento de un objeto en el espacio en relación a sí mismo, utilizando } \\
\text { los conceptos de izquierda-derecha, adelante-atrás, arriba-abajo, cerca-lejos y próximo- } \\
\text { lejano. }\end{array}$ \\
\hline Matemática & $\begin{array}{l}\text {-Elaborar y utilizar instrumentos y estrategias personales de cálculo mental y medida, } \\
\text { así como procedimientos de orientación espacial en contextos de resolución de } \\
\text { problemas, decidiendo, en cada caso, las ventajas de su uso y valorando la coherencia } \\
\text { de los resultados. } \\
\text {-Medición con instrumentos y estrategias no convencionales. } \\
\text {-Expresar correctamente la localización de un objeto en el espacio. } \\
\text {-Utilización de instrumentos de dibujo y programas informáticos para la construcción y } \\
\text { exploración de formas geométricas. } \\
\text {-Formulación de razonamientos y argumentaciones sobre la validez de una solución } \\
\text { identificando, en su caso, los errores. } \\
\text {-Colaboración activa y responsable en el trabajo en equipo, manifestando iniciativa para } \\
\text { resolver problemas que implican la aplicación de los contenidos estudiados. }\end{array}$ \\
\hline $\begin{array}{l}\text { Historia, } \\
\text { Geografía } \\
\text { Ciencias } \\
\text { Sociales }\end{array}$ & $\begin{array}{l}\text { Métodos Naturales: } \\
\text {-El sol, a través del cual podemos determinar los puntos cardinales. } \\
\text {-Métodos de orientación para el hemisferio sur. } \\
\text {-Métodos de orientación para el hemisferio norte. } \\
\text { Métodos Artificiales: } \\
\text {-El mapa, como representación gráfica de un espacio en un plano a escala. }\end{array}$ \\
\hline
\end{tabular}


-El plano, como representación gráfica de un espacio con la diferencia que éstos muestran más detalles de la realidad que los mapas.

-La brújula, que consiste en una caja rellena de líquido en cuyo interior se encuentra una aguja imantada que gira libremente sobre un soporte vertical y que siempre indica donde se encuentra el norte magnético.

-EI GPS que actúa a través de satélites, pero cuyo mayor inconveniente es que en espacios cerrados no recibe correctamente las señales.

-Uso de croquis, planos y mapas.

-Representación a escala de espacios conocidos.

-Diferentes representaciones sobre un mismo espacio (planos, fotografías aéreas y otros medios tecnológicos).

-Planificación de itinerarios.

-Localización en diferentes representaciones cartográficas de elementos relevantes de geografía física y política del mundo.

\begin{tabular}{ll}
\hline Educación & -Actividades de ubicación espacial y temporal como derecha-izquierda, adelante-atrás, \\
Física y Salud & adentro-afuera, entre, al lado, antes, durante, después, rápido y lento. \\
& -Orientación espacio-tiempo. \\
& -Resolución de problemas en relación al espacio y tiempo, trabajo de puntos cardinales. \\
& -Juegos de pistas o búsqueda del tesoro, trabajo de croquis y planos. \\
& -Deportes individuales: Orientación Terrestre, implementación deportiva, juegos de \\
& iniciación y familiarización con el deporte. \\
& -Cálculo de coordenadas geográficas y rectangulares. \\
& -Práctica de identificación de curvas de nivel. \\
& -Manejo de la brújula. \\
& -Carrera de orientación es espacios naturales: parques, alamedas, plazas, etc. \\
& -Planificación, organización y ejecución de un evento deportivo de Orientación Terrestre.
\end{tabular}

Fuente: elaboración de los autores.

A partir de estas ideas, se propone una relación entre los contenidos expuestos en los programas escolares y la metodología para el trabajo de orientación en la escuela desde los niveles de aprendizaje expuestos en este trabajo. En la Tabla 3, se presenta la relación de cada nivel de aprendizaje con los contenidos por materia: 
Tabla 3

Relación entre los niveles de aprendizaje y contenidos por materia.

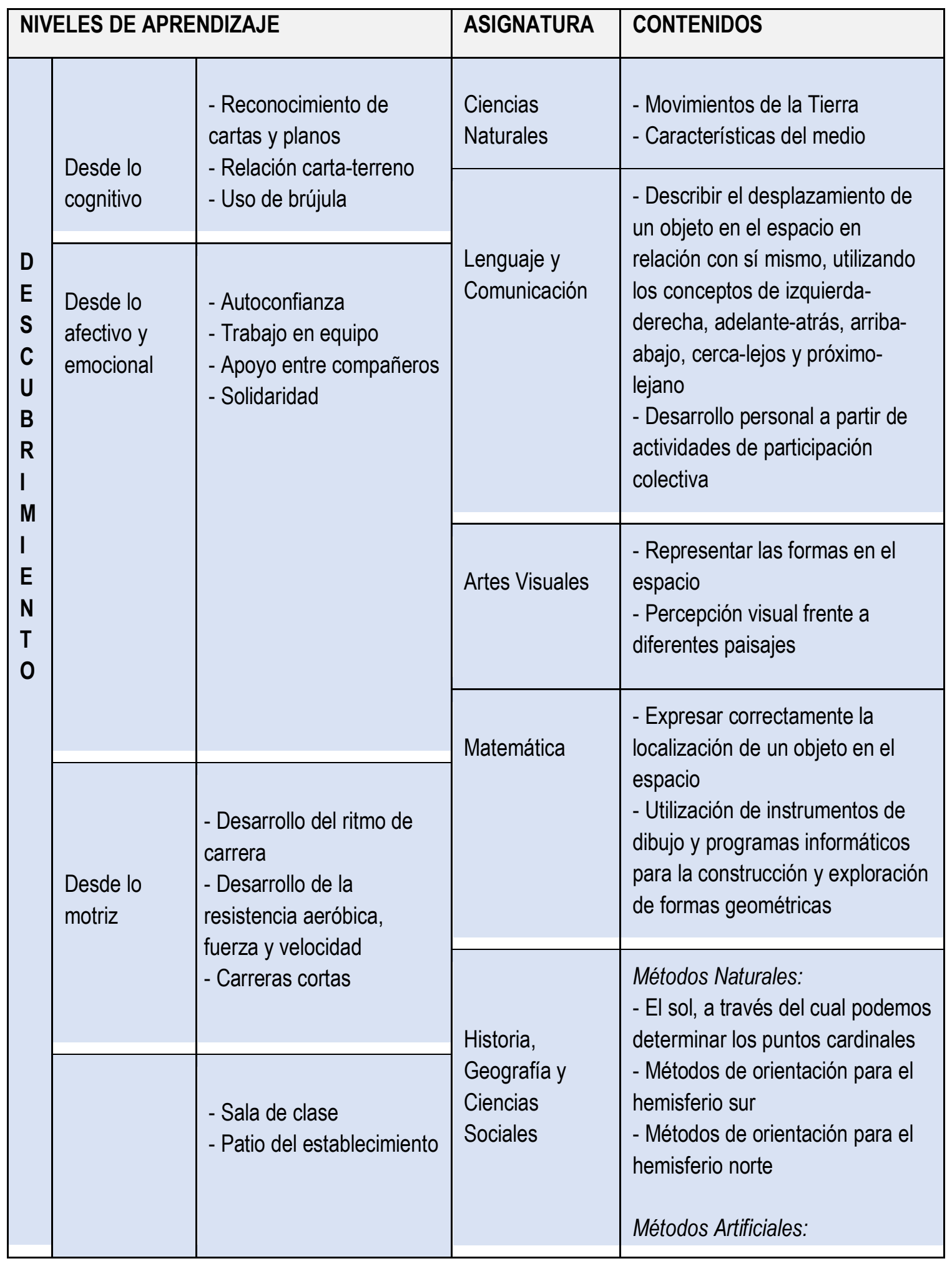




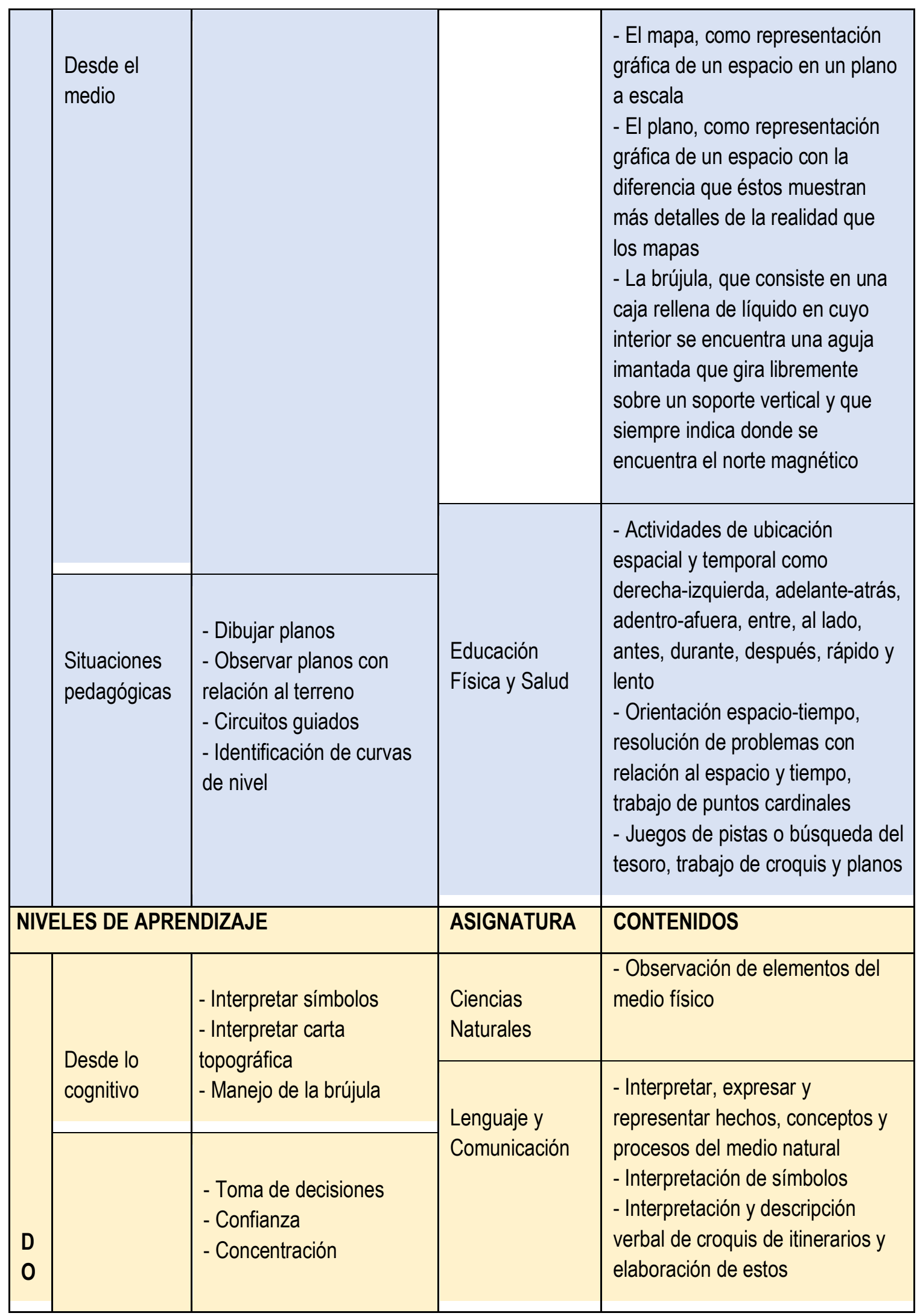




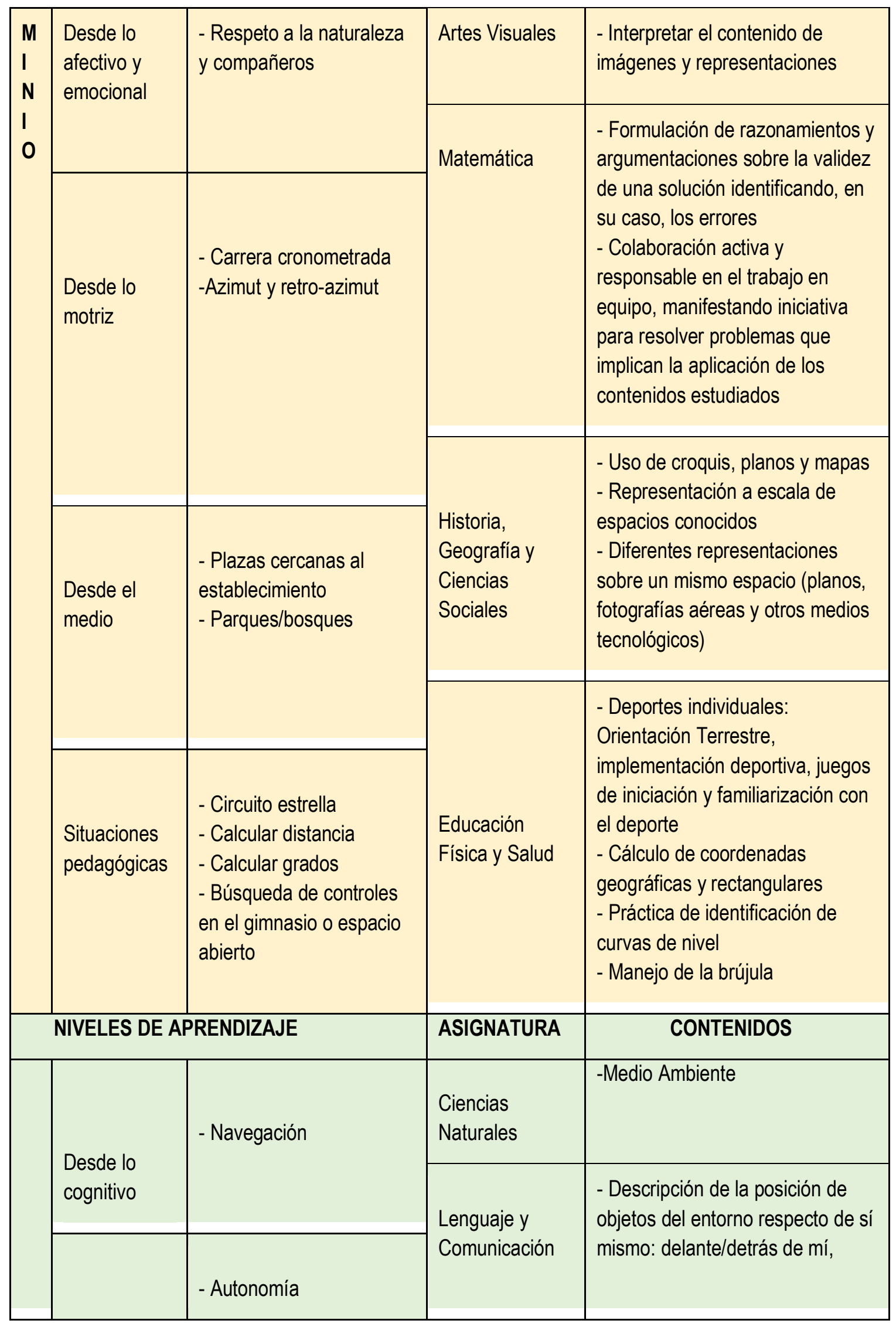




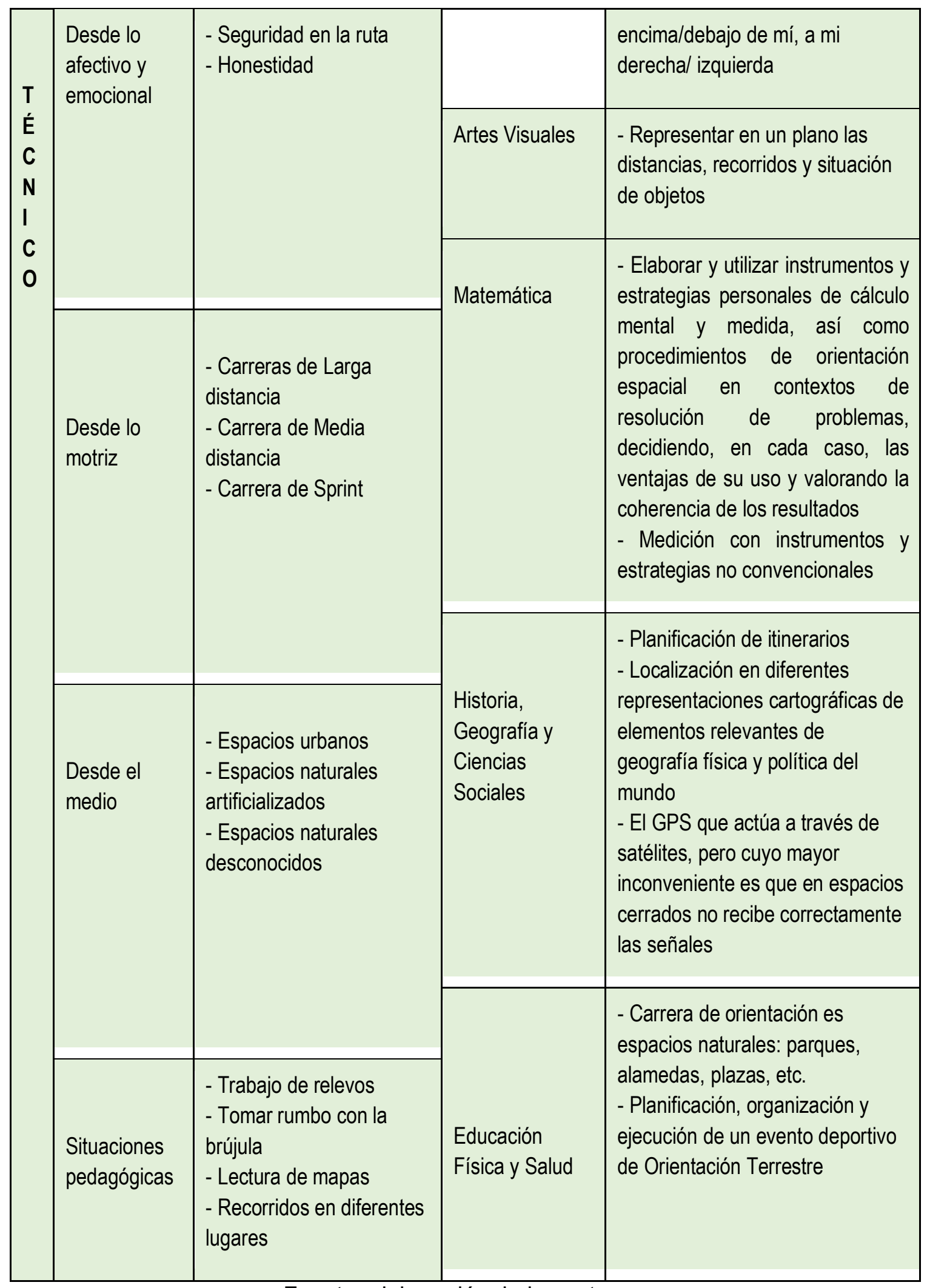

Fuente: elaboración de los autores. 


\section{Consideraciones finales}

Las posibilidades de crecimiento en los espacios naturales son indiscutiblemente considerables a partir de las evidencias bibliográficas, entendiendo que en la naturaleza están todas las respuestas a las grandes preguntas de la humanidad. De acuerdo a Fernández, Ruiz, Cortés y Pascual (1993), Sanhueza-Tsutsumi, González y Gutiérrez (2005), los sujetos sometidos a un entorno enriquecido presentan una mejor conducta exploratoria y una mayor capacidad de resolución de problemas, así como una evolución motora adaptativa más avanzada que aquellos sujetos que están en otros ambientes (artificializados). En este sentido, las vivencias cobran preeminencia en el momento que se le otorga significación, transformándose así en una relación dinámica que surge a partir de la información recogida a través de los órganos sensoriales y el sentido que le entrega su interpretación, vale decir, según Schütz (1993), que la construcción de este sentido es multidimensional y compleja, lo que entregaría algún impulso por establecer la comunicación-relación entre educación, interdisciplina y orientación. Junto con eso, las formas de relación ser humano-naturaleza, expresadas por medio de prácticas como la orientación deportiva, deben ser comprendidas desde la complejidad, de tal manera de no separar o reducir los aspectos del ser humano a lo físico, biológico, social, cultural, síquico y/o espiritual (Morin, 2010), en tal sentido, esta práctica deportiva-educativa-recreativa, debe tender a favorecer la complejidad de las relaciones cuerpo-naturaleza-cultura-accióncognición.

En tal sentido, esta propuesta de progresión espacial y metodológica interdisciplinar está centrada en el avance gradual de la incorporación de información desde lo más elemental hasta lo más complicado, tomando en consideración los elementos más abstractos y globales hasta llegar a lo específico y puntual, reforzando en su desarrollo contenidos y conceptos transversales relativos a la autoconfianza, autoestima, afectividad, respeto por el medio ambiente y los semejantes, toma de decisiones y resolución de problemas, además de abordar los temas específicos de cada materia o asignatura.

Para alcanzar este propósito, se sugiere utilizar la técnica por indagación, en la que se presentan situaciones didácticas basadas, fundamentalmente, en la resolución de problemas. Según Casado (2010), para conseguir resultados en una actividad de estas características, es necesaria una explicación inicial y otras de carácter técnico entre los ejercicios antes de introducir cada actividad, vale decir, entregar explicaciones cortas y simples con objetivos claros acompañadas de ejercicios para que el contenido sea asimilado de buena manera.

De acuerdo con la información obtenida, es posible concluir que el desarrollo de actividades en el medio natural y, en particular la Orientación Deportiva, es beneficioso para la formación integral del educando, toda vez que a partir de su práctica se promueve el desarrollo de ciertas habilidades y conductas asociadas al entendimiento del fenómeno humano a partir de la integración de saberes.

En términos de la delimitación de la extensión del estudio, es posible indicar que la aplicación de resultados puede verse asociado a temas de cualquier índole, toda vez que el principio de flexibilidad presentado por la actividad permite adecuaciones sin perder el foco y objetivo de la misma. En ese mismo sentido, la extensión teórica y práctica, se 
presenta como un referencial concreto coligado al contexto escolar y sus posibilidades de desarrollo, superando el abordaje técnico para pasar a un tratamiento integral basado en aprendizajes de orden vivencial y definido.

\section{Referencias}

Bardin, L. (2011). Análise de conteúdo. São Paulo: Edições 70.

Bell, A. y Dyment, J. (2006). Grounds for Action: Promoting Physical Activity through School Ground Greening in Canada. Toronto: Evergreen. Recuperado de https://www.evergreen.ca/tools-publications/grounds-for-action-promoting-physicalactivity-through-school-ground-greeni/

Carvajal, Y. (2010). Interdisciplinariedad: desafío para la Educación Superior y la investigación. Revista Luna Azul, (31), 156-169. Recuperado de http://www.scielo.org.co/pdf/luaz/n31/n31a11.pdf

Casado, J. (2010). El deporte de orientación en el ámbito educativo: posibilidades didácticas del gimnasio. EmásF: Revista Digital de Educación Física, 2(7) 82-94. Recuperado de https://emasf.webcindario.com/El_deporte_de_orientacion_en_el_ambito_ educativo.pdf

Castillo, F. (2006). Escuela, motricidad y medio natural. Revista Ciencias de la Actividad Física, 8, 51-60.

Castillo, F. (2010). Transversalidad, interdisciplinariedad y aprendizaje, una tríada que emerge de la espacialidad. Revista educación física y deporte, 2(29), 279-286. Recuperado de http://aprendeenlinea.udea.edu.co/revistas/index.php/educacionfisicay deporte/article/view/8503

Castillo, F. y Cordero, M. (2017). Actividades en la naturaleza. Propuesta curricular para Educación Física. En Textos de Apoyo a la Docencia, $n^{\circ} 39$. Talca: Universidad Católica del Maule.

Ministerio de Educación del Gobierno de Chile. (2012). Decreto Supremo $n^{\circ} 433$. Santiago: Ministerio de Educación. Recuperado de https://www.leychile.cl/Navegar?idNorma=1047359\&idVersion=2012-12-19

Díaz, J. (2010). Educación Física e interdisciplinariedad: una relación cada vez más necesaria. Revista Tándem, Didáctica de la Educación Física, 33, 7-21. Recuperado de https://www.grao.com/es/producto/revista-tandem-033-abril-10-interdisciplinaridady-educacion-fisica

Engel, T. y Tolfo, D. (2009). Métodos de pesquisa. Porto Alegre: Universidade Federal do Rio Grande do Sul. Recuperado de http://www.ufrgs.br/cursopgdr/downloadsSerie/derad005.pdf

Escaravajal-Rodríguez, J. (2017). La orientación deportiva como medio interdisciplinar. Revista FEAFYS, 9(6), 885-908. Recuperado de 
http://www.trances.es/index.php?option=com_content\&view=article\&id=424:escaravaj al-rodriguez-jc-2017-la-orientacion-deportiva-como-medio-interdisciplinar-trances96885-908\&catid=94:noviembre-diciembre-2017\&ltemid=76

Fernández, V., Ruiz, S., Cortés, P. y Pascual, R. (1993). Impacto del enriquecimiento ambiental sobre el desarrollo cerebral. Interacciones sociales, nutricionales y sensoriales. Revista Chilena de Nutrición, 21, 7-18.

Follari, R. (2007). La interdisciplina en la Docencia. Polis: Revista académica de la Universidad Bolivariana, 16, 1-14. Recuperado de https://journals.openedition.org/polis/4586

Freire, H. (2011). Educar en verde. Ideas para acercar a niños y niñas a la naturaleza. Barcelona: Graó. Recuperado de https://www.grao.com/es/producto/educar-en-verde

Fuentealba, M., Marín, F., Castillo, F. y Roco, L. (2017). Análisis de la experiencia pedagógica Campamento EXPLORA Chile VA! Valorando la Biodiversidad Maulina. Revista Actualidades Investigativas en Educación, 17 (1), 1-25. doi: http://dx.doi.org/ 10.15517/aie.v17i1.27211

Henao, C., García, D., Aguirre, E., González, A., Bracho, R., Solorzano, J. y Arboleda, A. (2017). Multidisciplinariedad, interdisciplinariedad y transdisciplinariedad en la formación para la investigación en ingeniería. Revista Lasallista de Investigación, 14(1), 179-197. doi: http://dx.doi.org/10.22507/rli.v14n1a16

Julián, J. y Pinos, M. (2011). Unidades didácticas de segundo ciclo de primaria. Actividad: Orientación. Zaragoza: Departamento de Educación, Universidad, Cultura y Deporte del Gobierno de Aragón. Recuperado de http://efypaf.unizar.es/recursos/orientacion.html

Kellert, S. (2005). Nature and Childhood Development. En Building for Life: Designing and understanding the human-nature connection. Washington, D.C.: Island Press. Recuperado de https://bit.ly/2nw0fIP

Lenoir, Y. (1999). Interdisciplinarité. En J. Houssaye (Coord.). Questions pédagogiques. Encyclopédie historique (pp. 291-314). Paris: Hachette. Recuperado de https://www.amazon.fr/Questions-p\%C3\%A9dagogiques-Encyclop\%C3\%A9diehistorique-Houssaye/dp/2011704960

Llano, L., Gutiérrez M., Stable, A., Núñez, M., Masó, R. y Rojas, B. (2016). La interdisciplinariedad: una necesidad contemporánea para favorecer el proceso de enseñanza aprendizaje. Revista Medisur, 14(3), 320-327. Recuperado de http://scielo.sld.cu/scielo.php?script=sci_arttext\&pid=S1727897X2016000300015

Longchamp-Ges, N., Escalona-Vásquez, I. y Céspedes-Quiala, A. (2018). La interdisciplinariedad: algunas consideraciones desde la carrera de Licenciatura en Educación Preescolar. Revista Maestro y Sociedad, (4), 178-185. Recuperado de https://bit.ly/2nIAhYK 
Marimon, M. y Martos, L. (2014). ¿Cómo gestionamos la subjetividad? En Ó. Rull. Redacción especializada. Barcelona: Universitat Pompeu Fabra.

McMillan, J. y Schumacher, S. (2005). Investigación Educativa. Una introducción conceptual. ( $5^{\circ} \mathrm{ed}$ ). Madrid: Pearson Educación. Recuperado de https://bit.ly/2nwPNRI

McNeill, C., Cory-Wrigth, J. y Renirew T. (2006). Carreras de Orientación. Guía de aprendizaje. Barcelona: Paidotribo. Recuperado de https://www.amazon.es/CARRERAS-ORIENTACI\%C3\%93N-aprendizaje-ColorDeportes/dp/8480198818

Miller, F. (1971). Principios y servicios de orientación escolar. Madrid: Magisterio Español. Recuperado de https://bit.ly/2mKadWU

Morin, E. (2010). Ciência com consciência. Bertrand: Rio de Janeiro.

Muñoz, J. (2010). El cross de orientación. Aplicaciones y posibilidades educativas. Sugerencias para su organización. Revista Temas para la Educación, 10, 1-9. Recuperado de https://www.feandalucia.ccoo.es/docu/p5sd7449.pdf

Paula-Acosta, C., Pérez-López, J. y Sierra-Socorro, J. (2019). La educación ambiental con enfoque integrador. Una experiencia en la formación inicial de profesores de matemática y física. Revista electrónica Educare, 23 (1), 1-22. doi: http://dx.doi.org/10.15359/ree.23-1.10

Pimentel, G., Castillo, F., Fernandes, A., Noda, L., Da Silva, L. y Dos Santos, S. (2017). Atividades alternativas na Educação Física escolar. Revista Educação Física UNIFAFIBE/SP, 5, 176-196. Recuperado de http://unifafibe.com.br/ revistasonline/arquivos/revistaeducacaofisica/sumario/56/30082017172544.pdf

Posada, R. (2004). Formación Superior basada en competencias, interdisciplinariedad y trabajo autónomo del estudiante. Revista Iberoamericana de Educación, 35 (1), 1-33. Recuperado de https://rieoei.org/RIE/article/view/2870/3814

Rivero, S., López-Huertas, M. y Pérez, M. (2013). La interdisciplinariedad de la ciencia y la organización del conocimiento en los sistemas de gestión de información curricular. Revista Cubana de Información en Ciencias de la Salud, 24 (3), 354-367. Recuperado de http://scielo.sld.cu/scielo.php?script=sci_arttext\&pid=S2307-21132013000300011

Sanhueza-Tsutsumi, M., González, X. y Gutiérrez, R. (2005). Estudio Exploratorio: Relación de la conciencia corporal y la estructuración témporo-espacial en el aprendizaje de la lectoescritura en niños de primer año básico. Revista Iberoamericana de Educación, 35 (5), 1-15. Recuperado de http://www.scielo.org.co/scielo.php?script=sci_ nlinks\&ref=000090\&pid=S0123-4226200800010000800016\&lng=pt

Schütz, A. (1993). La construcción significativa del mundo social. Barcelona: Paidós.

Sicilia, A. y Rivadeneira, M. (1998). Unidades Didácticas para Secundaria. Barcelona: INDE.

Silva, E. y Muszkat, E. (2005). Metodologia da pesquisa e elaboração de dissertação (4a ed.). Florianópolis: Universidade Federal de Santa Catarina. Recuperado de https://projetos.inf.ufsc.br/arquivos/Metodologia_de_pesquisa_e_elaboracao_de_tese s_e_dissertacoes_4ed.pdf 
Sotolongo, P. y Delgado, C. (2016). La complejidad y el diálogo transdisciplinario de saberes. Trans-Pasando Fronteras: 10, 11-24. doi: https://doi.org/ 10.18046/retf.i10.2631

Torres, J. (1996). Globalización e interdisciplinariedad: El currículum integrado. Madrid: Morata.

Van del Linde, G. (2007). ¿Por qué es importante la interdisciplinariedad en la educación superior? Cuadernos de Pedagogía Universitaria, 4 (8), 11-12. Recuperado de http://cuaderno.pucmm.edu.do/index.php/cuadernodepedagogia/article/view/68/67

Varela, F. (2000). El Fenómeno de la Vida. Santiago de Chile: Dolmen.

Wells, N. (2000). At home with nature: Effects of "greenness" on children's cognitive functioning. Journal Environment and Behavior, 32 (6), 775-795. doi: https://doi.org/10.1177/00139160021972793 